\title{
Desenvolvimento do Pensamento Computacional na Ciência da Computação: uma Questão de Gênero?
}

\author{
Tamara C. Ferreira ${ }^{1}$, Joslaine Cristina J. de Freitas ${ }^{1}$, \\ Marcos Wagner S. Ribeiro ${ }^{1}$, Eliane Raimann ${ }^{2}$ \\ ${ }^{1}$ Instituto de Ciências Exatas e Tecnológicas \\ Universidade Federal de Goiás - Regional Jataí (UFG/ Regional Jataí) \\ 758101-615 - Jataí - GO - Brazil \\ ${ }^{2}$ Instituto de Educação, Ciência e Tecnologia \\ Instituto Federal de Goiás (IFG) - Jataí, GO - Brazil \\ \{mhara92cinder, joslaine, marcoswagnersouza, elianeraimann\}@gmail.com
}

\begin{abstract}
Computational Thinking is understood as the process of thinking of the formulation of a problem and in the expression of its solution so that it can be effectively effected. The objective of this work is to analyze the level of PC development between men and women when they enter the computer faculty and also during the course, in order to indicate if the development of the PC can influence the entrance in Computer courses and if it really exists an increase in $C P$ index in training time. For this, a questionnaire was developed based on the five dimensions of the CP (abstraction, generalization, modularity, algorithm and decomposition). The results showed that performance is relatively equal between men and women.
\end{abstract}

Resumo. Entende-se por Pensamento Computacional - PC o processo de pensamento da formulação de um problema e na expressão de sua solução de forma que se possa efetivamente efetuá-lo. O objetivo deste trabalho é analisar o nível de desenvolvimento do PC entre homens e mulheres quando entram na faculdade de computação e também durante o curso, com o intuito de apontar se o desenvolvimento do PC pode influenciar no ingresso em cursos de Computação e se realmente existe um crescimento no índice do PC no tempo de formação. Para tanto, desenvolveu-se um questionário baseado nas cinco dimensões do PC (abstração, generalização, modularidade, algoritmo e decomposição). Os resultados mostraram que o desempenho é relativamente igual entre homens e mulheres.

\section{Introdução}

No ano de 2007, a Sociedade Brasileira de Computação - SBC realizou o primeiro congresso Women in Information Technology - WIT para discutir assuntos referentes ao gênero nos cursos e na área tecnológica. Após essa iniciativa, [Maciel and Bim 2017] aponta que "uma série de projetos tem sido executados nas instituições adeptas ao programa". Atualmente, a quantidade de ingressantes em cursos de tecnologia está sendo muito analisada em diversos países e podem auxiliar no ingresso e/ou permanência das mulheres nos cursos de Computação, [Louzada et al. 2014]. 
VIII Congresso Brasileiro de Informática na Educação (CBIE 2019)

Anais do XXV Workshop de Informática na Escola (WIE 2019)

Segundo o Instituto Nacional de Estudos e Pesquisas Educacionais Anísio Teixeira - INEP, vinculado ao Ministério da Educação - MEC, as mulheres representam cerca de $15 \%$ dos matriculados nos cursos da área da tecnologia. Contudo, mesmo com o baixo ingresso e/ou interesse das mulheres em cursos de Computação, em contrapartida, as mulheres representam 57,2\% dos matriculados no ensino superior. Nesse contexto, diversos trabalhos vem sendo realizados com o intuito de disseminar a área de Computação às mulheres. Nos trabalhos de [HALMANN et al. 2005, Pinheiro et al. 2017, Santos et al. 2017] foram propostos alguns métodos de promoção da área de Computação utilizando o ensino de programação, o qual auxilia na disseminação e/ou aplicação do Pensamento Computacional - PC.

O PC baseia-se nos conceitos que são fundamentais para a Ciência da Computação e envolve o processamento de forma sistemática e eficiente de informações e tarefas [Wing 2006]. Também confronta o enigma da inteligência da máquina, analisando o que os humanos podem fazer melhor que os computadores e o que os computadores podem fazer melhor que os humanos [Wing 2008]. Nesse contexto, a autora afirma que o PC inclui uma gama de ferramentas mentais que refletem a amplitude do campo da Ciência da Computação. Porém, [Araujo et al. 2016], após realizarem um mapeamento sobre o PC, observaram a ausência de trabalhos com intuito de avaliar o PC no contexto de resolução de problemas, bem como as métricas utilizadas para garantir a proficiência em PC.

Contudo, analisando a crescente disseminação do PC às mulheres para promoção da área da Computação; o baixo índice de ingresso e interesse das mulheres à área de Computação; e o fato dos homens serem mais propensos a terem uma aula de programação de computadores do que as mulheres [Sales et al. 2014] - sendo que esse fator pode expressar o baixo interesse em seguir carreira em Ciência da Computação [Weisgram and Bigler 2006, Shashaani 1994], este trabalho pretende analisar o nível de desenvolvimento do PC entre homens e mulheres quando entram na faculdade de computação e durante o curso, com o intuito de apontar se o desenvolvimento do PC pode influenciar no ingresso em cursos de Computação e se realmente existe um crescimento no índice do PC no tempo de formação.

\section{Pesamento Computacional}

Umas das características do PC é que ele é baseado "no poder e nos limites de processos computacionais, sejam eles executados por um ser humano ou por uma máquina" [Wing 2006]. O PC está relacionado na maneira de um indivíduo conseguir resolver ou interpretar problemas, utilizando a máquina apenas como um recurso. Assim, a essência do PC está pensando como um cientista da Computação quando confrontado com um determinado problema.

No trabalho de [Atmatzidou and Demetriadis 2016], os autores citaram cinco dimensões centrais da estrutura conceitual mais ampla do PC que consiste em abstração, generalização, algoritmo, modularidade e decomposição. Tais dimensões podem contribuir na avaliação do nível do PC dos indivíduos, pois de acordo com [Wing 2006] o PC é uma habilidade fundamental e [Blikstein 2008], cita que o PC é uma importante habilidade para todos no século XXI. 
VIII Congresso Brasileiro de Informática na Educação (CBIE 2019)

Anais do XXV Workshop de Informática na Escola (WIE 2019)

\subsection{Abstração}

A abstração é um dos principais pilares na avaliação do PC [Wing 2006, Brennan and Resnick 2012], a qual permite fragmentar um problema em partes menores para facilitar a resolução. Nesse contexto, [Becker 2014] aponta que a abstração consiste na retirada de qualidades dos objetos a serem observados, algo que é análogo ao ouvirmos o som de um instrumento, tocarmos um sino, ou observarmos um carro em movimento. Tais qualidades quando retiradas das ações (ouvir, tocar, observar) ou dos objetos (instrumento, sino, carro) são todas observáveis.

De acordo com [Piva Jr and Freitas 2010], a habilidade cognitiva da associação de imagens à enunciados lidos, pode ser considerara uma forma de abstração. Uma das aplicações dessa estratégia seria por meio da utilização de textos, de modo que o leitor faça uma interpretação do conteúdo lido por meio de um desenho. Outra forma de avaliação apontada em seu trabalho é o inverso da estratégia anterior que consiste na análise de uma determinada figura ou ilustração, de modo que seja feito um texto que exprima o que foi visualizado. Neste caso, não é contada a erudição do estudante, mas a capacidade de reconhecer detalhes e as inter-relações existentes entre os elementos da figura ou ilustração.

\subsection{Generalização}

No trabalho de [Mattos 2001], o autor aponta a generalização como o resultado do processo de abstração da solução do caso em questão, onde o número-de-passos, númerode-variáveis e número-de-constantes são métricas utilizadas para facilitar na definição do perfil deste caso e usadas na fase de seleção dos melhores casos à um determinado contexto. O processo de generalização é decorrente do processo de abstração, partindo da caracterização de um determinado contexto. Conforme [Vygotsky et al. 2008], a generalização é um ato do pensamento totalmente semântico que reflete a realidade, de forma que difere-se bastante de como esta é refletida nas sensações e nas percepções imediatas. Pode-se dizer que o processo de abstração está ligado a um deslocamento realizado pelo sujeito, onde por meio da abstração, ele é capaz de dissociar e generalizar determinados aspectos de uma determinada realidade [Taxa et al. 2001].

\subsection{Modularidade}

A definição do conceito de modularidade difere-se da modularização, porém elas se interrelacionam. No trabalho de [Ulrich 1994], o autor aponta que modularidade é a independência dos componentes e a propriedade de um projeto que permite a padronização e a permutação. A modularidade surge da maneira como um produto é fisicamente dividido em componentes. Uma característica da modularidade é o grau em que as interações entre os componentes físicos estão confinadas para a função do produto [Ulrich 1994]. A divisão em módulos de um determinado produto para se trabalhar as partes, onde a interação dos módulos formam o produto final, é denominada modularidade.

\subsection{Algoritmo}

De acordo com o dicionário Michaelis, algoritmo é um conjunto das regras de operação (conjunto de raciocínios), cuja aplicação permite resolver um problema enunciado por meio de um número finito de operações. Pode ser traduzido em um programa executado 
VIII Congresso Brasileiro de Informática na Educação (CBIE 2019)

Anais do XXV Workshop de Informática na Escola (WIE 2019)

por um computador, detectável nos mecanismos gramaticais de uma língua ou no sistema de procedimentos racionais finito utilizado em outras ciências para resolução de problemas semelhantes. Algoritmo pode ser caracterizado como uma sequências de passos para chegar a um determinado resultado e um exemplo simples é a receita de um bolo, visto que existe uma série de ingredientes e procedimentos (passos) necessários para conseguir chegar ao bolo (resultado).

\subsection{Decomposição}

A decomposição está relacionada com a separação de quantidades em unidades menores para chegar ao resultado final [Correa and Moura 1997]. A decomposição na Matemática e na Computação trabalham na mesma linha, sendo a divisão de um problema para trabalhar as partes do mesmo.

\section{Metodologia}

Com o objetivo de desenvolver um método de avaliação do nível do PC foi desenvolvido um questionário que contém as cinco dimensões do PC (abstração, generalização, modularidade, algoritmo e decomposição). O questionário foi aplicado aos ingressantes e veteranos do curso de Bacharel em Ciência da Computação - BCC da Universidade Federal de Goiás - UFG/Regional Jataí localizada na cidade de Jataí. Contudo, para conseguir avaliar o nível de desenvolvimento do PC dos alunos, partiu-se dos conceitos de cada dimensão do PC aplicada no questionário, com o intuito de construir uma base sólida para que os dados obtidos exprimam um caso real.

\subsection{Questionário}

O questionário contém 9 questões objetivas e 1 questão dissertativa, sendo que as nove primeiras questões contém cinco alternativas (A, B, C, D e E). As Figuras 1, 2, 3 e 4 mostram o questionário usado para análise do desenvolvimento do $\mathrm{PC}$, onde das 10 questões contidas no questionário, cada uma equivale a $10 \%$ do questionário total.

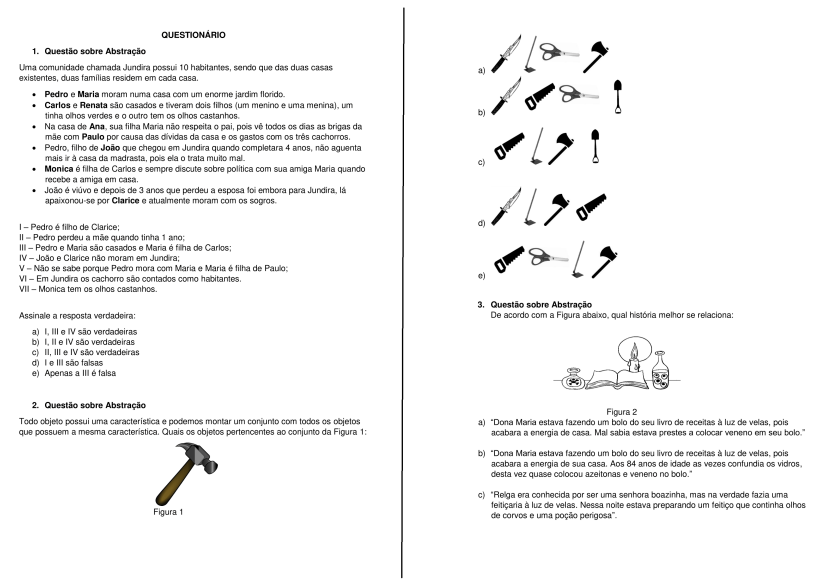

Figura 1. Questionário aplicado ao curso de BCC - página 1 e página 2

As questões 1 á 4 (40\%), pretendem delinear o desenvolvimento de abstração; as questões 5 e 6 (20\%), pretendem delinear sobre o desenvolvimento da generalização; as 
VIII Congresso Brasileiro de Informática na Educação (CBIE 2019)

Anais do XXV Workshop de Informática na Escola (WIE 2019)

questões 7 e 8 (20\%) pretendem delinear o desenvolvimento de modularidade; a questão 9 (10\%) pretende delinear o desenvolvimento de algoritmo; e a questão $10(10 \%)$ pretende delinear o desenvolvimento de decomposição do participante.

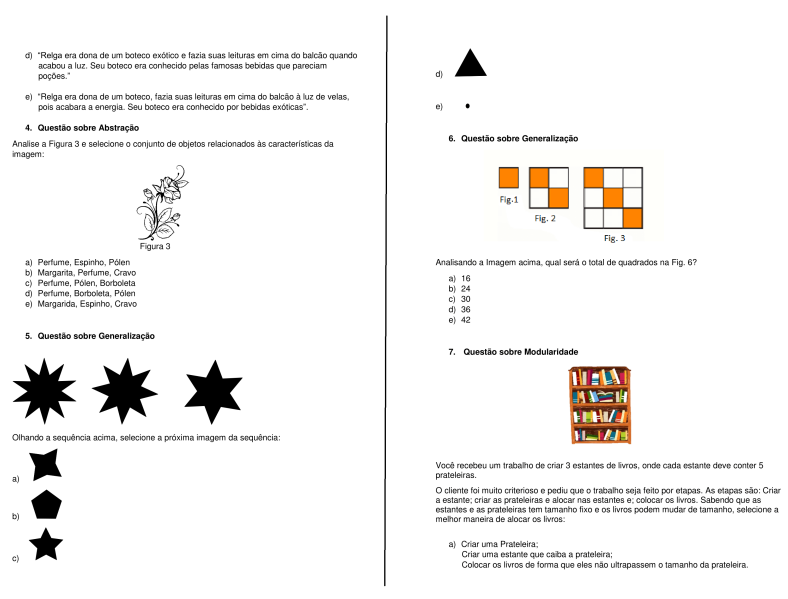

Figura 2. Questionário aplicado ao curso de BCC - página 3 e página 4

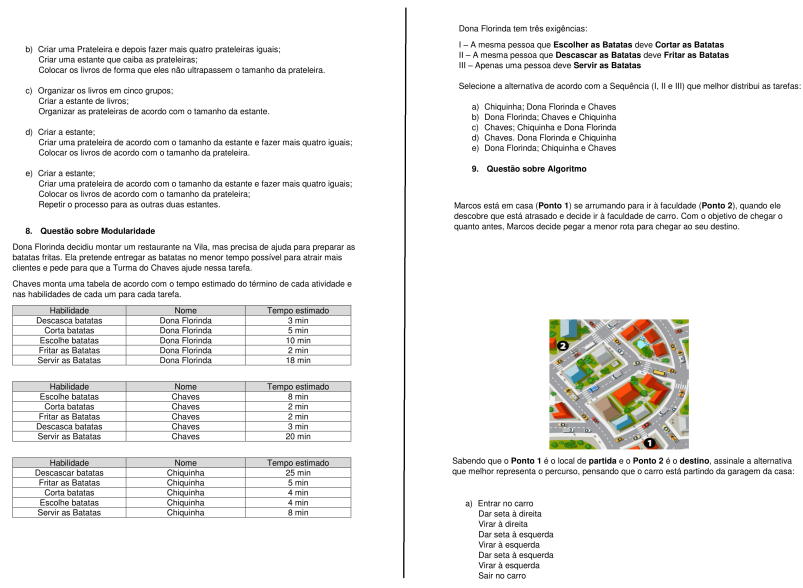

Figura 3. Questionário aplicado ao curso de BCC - página 5 e página 6

\section{Resultados e Discussão}

O questionário foi respondido pelo total de 51 pessoas, sendo 37 alunos ingressantes do curso de BCC ( 5 mulheres e 32 homens) e 14 alunos veteranos do curso de BCC (4 mulheres e 10 homens) da UFG/Regional Jataí. Os dados foram organizados em uma tabela que possibilitou a geração dos gráficos para comparação dos resultados, como mostrado no gráfico contido na Figura 5.

De acordo com as respostas das 4 questões de abstração (40\%), os discentes ingressantes do sexo masculino tiveram uma porcentagem de 25,9\% (14 acertos na primeira questão; 29 acertos na segunda questão; 25 acertos na terceira questão; e 15 acertos na quarta questão) de desenvolvimento em abstração, enquanto os discentes do sexo feminino tiveram uma porcentagem de 30\% (3 acertos na primeira questão; 2 acertos na segunda questão; 5 acertos na terceira questão; e 5 acertos na quarta questão). Entre os 
VIII Congresso Brasileiro de Informática na Educação (CBIE 2019)

Anais do XXV Workshop de Informática na Escola (WIE 2019)
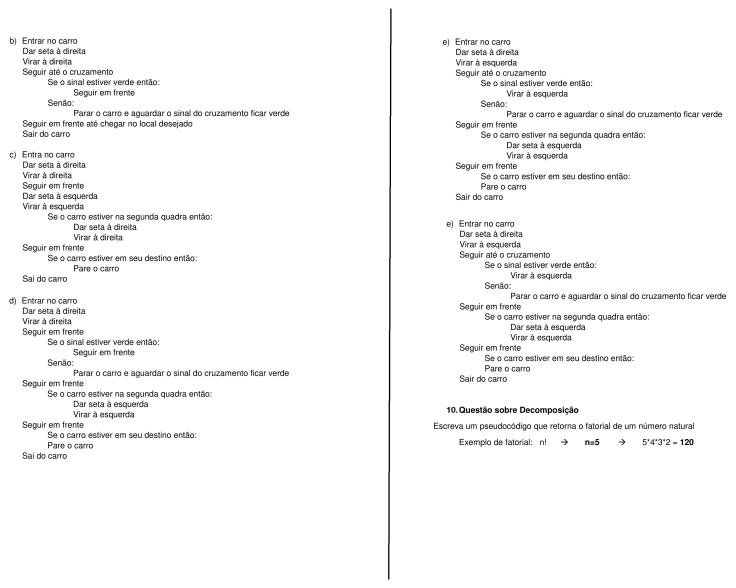

Figura 4. Questionário aplicado ao curso de BCC - página 7 e página 8

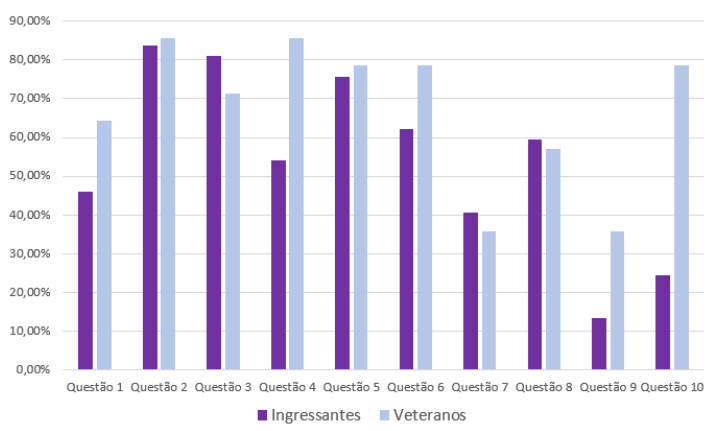

Figura 5. Porcentagem de acertos entre os ingressantes e veteranos do curso de BCC

veteranos, os discentes do sexo masculino tiveram uma porcentagem de $28 \%$ (6 acertos na primeira questão; 8 acertos na segunda questão; 6 acertos na terceira questão; e 8 acertos na quarta questão) de desenvolvimento em abstração, enquanto os discentes do sexo feminino tiveram uma porcentagem de 38\% (3 acertos na primeira questão; 4 acertos na segunda questão; 4 acertos na terceira questão; e 4 acertos na quarta questão).

De acordo com as respostas das 2 questões de generalização (20\%), os discentes ingressantes do sexo masculino tiveram uma porcentagem de 15,9\% (28 acertos na quinta questão e 23 acertos na sexta questão) de desenvolvimento em generalização, enquanto os discentes do sexo feminino tiveram uma porcentagem de $15 \%$ (3 acertos na quinta questão e 4 acertos na sexta questão). Entre os veteranos, os discentes do sexo masculino tiveram uma porcentagem de $17 \%$ ( 8 acertos na quinta questão e 9 acertos na sexta questão) de desenvolvimento em generalização, enquanto os discentes do sexo feminino tiveram uma porcentagem de $12,5 \%$ ( 3 acertos na quinta questão e 2 acertos na sexta questão).

De acordo com as respostas das 2 questões de modularidade (20\%), os discentes ingressantes do sexo masculino tiveram uma porcentagem de 9,7\% (11 acertos na sétima questão e 20 acertos na oitava questão) de desenvolvimento em modularidade, enquanto os discentes do sexo feminino tiveram uma porcentagem de 12\% (4 acertos na sétima questão e 2 acertos na oitava questão). Entre os veteranos, os discentes do sexo masculino tiveram uma porcentagem de 8\% (3 acertos na sétima questão e 5 acertos na oitava 
VIII Congresso Brasileiro de Informática na Educação (CBIE 2019)

Anais do XXV Workshop de Informática na Escola (WIE 2019)

questão) de desenvolvimento em modularidade, enquanto os discentes do sexo feminino tiveram uma porcentagem de 13\% ( 2 acertos na sétima questão e 3 acertos na oitava questão).

De acordo com as respostas da questão de algoritmo (10\%), os discentes ingressantes do sexo masculino tiveram uma porcentagem de 1,6\% (5 acertos na nona questão) de desenvolvimento em algoritmo, enquanto nenhuma das 5 ingressantes do sexo feminino acertaram a questão referente à algoritmo. Entre os veteranos, os discentes do sexo masculino tiveram uma porcentagem de 3\% (3 acertos na nona questão) de desenvolvimento em algoritmo, enquanto os discentes do sexo feminino tiveram uma porcentagem de 5\% (2 acertos na nona questão).

De acordo com as respostas da questão de decomposição (10\%), os discentes ingressantes do sexo masculino tiveram uma porcentagem de $2,5 \%$ (8 acertos na décima questão) de desenvolvimento em decomposição, enquanto os discentes do sexo feminino tiveram uma porcentagem de $2 \%$ ( 1 acerto na décima questão). Já entre os veteranos, os discentes do sexo masculino tiveram uma porcentagem de $8 \%$ (8 acertos na décima questão) de desenvolvimento em decomposição, enquanto os discentes do sexo feminino tiveram uma porcentagem de 7,5\% (3 acertos na décima questão).

Para apontar o desenvolvimento em PC baseado nas dimensões que foram adotadas neste trabalho, foi gerada uma tabela com a soma das porcentagens com a finalidade de apontar o desenvolvimento total do PC, como mostrado na Tabela 1. Analisando os dados de acordo com a Tabela 1, o PC é estimulado durante o curso, o que proporciona o aumento do nível do PC em relação aos discentes ingressantes e os veteranos. Nota-se que o nível de desenvolvimento PC das discentes do sexo feminino cresceu em $18 \%$ enquanto o PC dos discentes do sexo masculino aumentou cerca de $8 \%$, o que demonstra um crescente aumento entre o PC das discentes do sexo feminino em comparação aos discentes do sexo masculino.

O percentual total de desempenho no questionário das discentes veteranas do sexo feminino foi de $76,5 \%$, onde este desempenho superior, pode estar relacionado com o esforço que as mulheres fazem para conseguir quebrar as barreiras impostas pela sociedade para ingressar em um curso que emprega em sua maioria homens. Segundo [Guimond and Roussel 2001], alguns fatores podem levar ao baixo desempenho em testes de habilidades e isso pode acontecer quando as mulheres sentem-se inferiorizadas num determinado ambiente.

\section{Tabela 1. Porcentagem de desenvolvimento do PC do curso de Ciência da Computação}

\begin{tabular}{ccc} 
& Feminino & Masculino \\
\hline Ingressantes & $58 \%$ & $55,6 \%$ \\
\hline Veteranos & $76,5 \%$ & $63,5 \%$ \\
\hline
\end{tabular}

Em algoritmo, os discentes do sexo masculino entram no curso com um baixo índice de desenvolvimento e as discentes do sexo feminino não possuíram nenhum índice de desenvolvimento, o que reforça a afirmação feita por [Weisgram and Bigler 2006] sobre a oportunidade dos meninos terem aulas de programação ser superior em relação as meninas. Em decomposição os discentes apresentaram um desempenho relativamente 
VIII Congresso Brasileiro de Informática na Educação (CBIE 2019)

Anais do XXV Workshop de Informática na Escola (WIE 2019)

igual, como afirma [Spinelli and SOUZA 2005] os alunos do ensino médio normalmente já possuem conhecimento sobre a decomposição dos números, porém, devido ao desconhecimento de escrita em pseudocódigo pode ter ocasionado o baixo índice. Contudo, a Figura 6, mostra que o percentual de desenvolvimento dos ingressantes é relativamente igual.

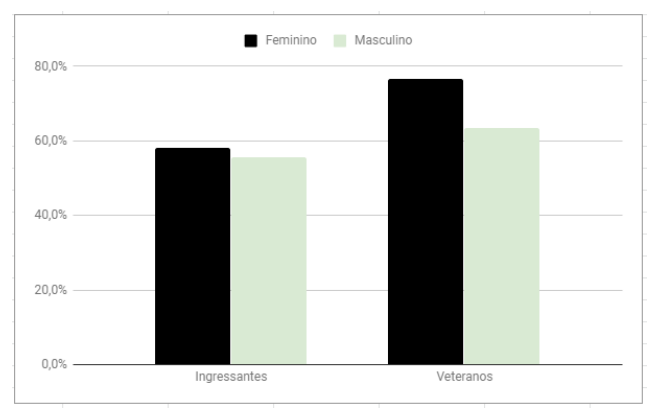

Figura 6. Porcentagem de desenvolvimento do PC entre os ingressantes e veteranos do curso de BCC

\section{Conclusão}

Com a avaliação do PC, pode-se concluir que homens que ingressam nos cursos de Computação possuem um melhor desenvolvimento em Abstração e Generalização. Contudo na análise geral é mostrado um desempenho relativamente igual entre os ingressantes de ambos os sexos. Além disto, a partir deste estudo, pode-se avaliar o PC de mulheres e homens e a partir dos resultados, apontar a necessidade de implantar medidas para desenvolver o PC antes do ingresso nos cursos de Computação, bem como mostrar que existe um aumento do nível do desenvolvimento do PC durante o curso de BCC. Para trabalhos futuros, pretende-se aplicar o questionário para um número maior de estudantes em diferentes níveis para que se consiga fazer comparativos do desenvolvimento do PC e criar uma metodologia que possa avaliar o PC desde a infância até a conclusão do curso superior.

\section{Referências}

Araujo, A. L., Andrade, W., and Guerrero, D. (2016). Um mapeamento sistemático sobre a avaliaçao do pensamento computacional no brasil. In Anais dos Workshops do Congresso Brasileiro de Informática na Educação, volume 5, page 1147.

Atmatzidou, S. and Demetriadis, S. (2016). Advancing students' computational thinking skills through educational robotics: A study on age and gender relevant differences. Robotics and Autonomous Systems, 75:661-670.

Becker, F. (2014). Abstração pseudo-empírica e reflexionante: Significado epistemológico e educacional. Schème: Revista Eletrônica de Psicologia e Epistemologia Genéticas. Marília, SP. Vol. 6, n. nesp (2014), p. 104-128.

Blikstein, P. (2008). O pensamento computacional e a reinvenção do computador na educação. Education \& Courses.

Brennan, K. and Resnick, M. (2012). New frameworks for studying and assessing the development of computational thinking. In Proceedings of the 2012 annual meeting 
VIII Congresso Brasileiro de Informática na Educação (CBIE 2019)

Anais do XXV Workshop de Informática na Escola (WIE 2019)

of the American Educational Research Association, Vancouver, Canada, volume 1, page 25 .

Correa, J. and Moura, M. L. S. d. (1997). A solução de problemas de adição e subtração por cálculo mental. Psicologia: reflexão e crítica, 10(1).

Guimond, S. and Roussel, L. (2001). Bragging about one's school grades: Gender stereotyping and students' perception of their abilities in science, mathematics, and language. Social psychology of education, 4(3-4):275-293.

HAlMANN, A. L., BONIlla, M. H. S., Martins, É. d. C., PEREIRA, G. d. d. S., Oliveira, H. L., Costa, H. M., Silva, L. B. d., SANTOS, P., CASTRO, T., BARRETO, U., et al. (2005). Construção coletiva do saber: uma vivência na faced/ufba. Anais do VI Cinform. Salvador.

Louzada, C. S., Gomes, W. F., Nunes, M., Salgueiro, E. M., Andrade, B. T., and Lima, P. (2014). Um mapeamento das publicações sobre o ingresso das mulheres na computação. In CLEI 2014: Conferência Latino-americana em Informática-VI Congresso da Mulher Latino-americana na Computação. Montevidéu.

Maciel, C. and Bim, S. A. (2017). Programa meninas digitais-ações para divulgar a computação para meninas do ensino médio. Anais do Computer on the Beach, pages 327-336.

Mattos, M. M. (2001). Construção de abstrações em lógica de programação. In Anais do XXI Congresso da Sociedade Brasileira de Computação, Fortaleza.

Pinheiro, A., Franco, J., and Leite, J. (2017). Desenvolvimento do pensamento computacional e discussões sobre representação feminina na computação: um estudo de caso. In Anais dos Workshops do Congresso Brasileiro de Informática na Educação, volume 6, page 1111 .

Piva Jr, D. and Freitas, R. L. (2010). Estratégias para melhorar os processos de abstração na disciplina de algoritmos. In Brazilian Symposium on Computers in Education (Simpósio Brasileiro de Informática na Educação-SBIE), volume 1.

Sales, A., Calado, B., Silva, D. R., Mattos, G., and Moreira, J. A. (2014). Dificuldades para o ingresso e permanência na ciência e engenharia da computação: um olhar feminino. 18th Redor-Universidade Federal Rural de Pernambuco-Recife.

Santos, C. P., Silva, D., Silveira, M., and Ferreira, G. (2017). Desafio de programaçao para meninas do ensino médio: Um relato de experiência. In Anais do Workshop de Informática na Escola, volume 23, page 137.

Shashaani, L. (1994). Gender-differences in computer experience and its influence on computer attitudes. Journal of Educational Computing Research, 11(4):347-367.

Spinelli, W. and SOUZA, M. H. (2005). Matemática. Ensino Médio. $1^{a}$ Edição. Nova Geração. São Paulo.

Taxa, F. d. O. S. et al. (2001). Problemas multiplicativos e processo de abstração em crianças na $3^{\mathrm{a}}$ série do ensino fundamental.

Ulrich, K. (1994). Fundamentals of product modularity. In Management of Design, pages 219-231. Springer. 
VIII Congresso Brasileiro de Informática na Educação (CBIE 2019)

Anais do XXV Workshop de Informática na Escola (WIE 2019)

Vygotsky, L. S. et al. (2008). Pensamento e linguagem.

Weisgram, E. S. and Bigler, R. S. (2006). Girls and science careers: The role of altruistic values and attitudes about scientific tasks. Journal of Applied Developmental Psychology, 27(4):326-348.

Wing, J. M. (2006). Computational thinking. Communications of the ACM, 49(3):33-35.

Wing, J. M. (2008). Computational thinking and thinking about computing. Philosophical transactions of the royal society of London A: mathematical, physical and engineering sciences, 366(1881):3717-3725. 